\section{Warum ich einen Arztroman schreibe?}

\section{E. Mosimann}

Warum nicht?

Als Chirurg kenne ich den Spitalalltag und auch den Betrieb im Operationssaal bestens, und immer noch scheinen Romane und TV-Serien rund um die Medizin ein grosses Publikum anzusprechen.

Zudem liegt der Hang zum Schreiben offenbar in der Familie, hat doch bereits mein Vater im Turnvereinheftli immer den Bericht über den Maibummel geschrieben.

\section{Was ich erreichen will?}

Gelesen werden und Erfolg haben, natürlich. Obschon das in der Schweiz nicht ganz so einfach ist. Es sei denn, man ist in den Medien schon präsent. Leute wie Thurnheer, Schawinski, Renato Tosio, Ralph Krüger haben es da schon etwas einfacher. Haben wahrscheinlich auch mehr zu sagen.

Zugegeben, die Affiche "Herzchirurg schreibt Arztroman" hat mir auch etwas geholfen.

Es ist der Autor, der sich vermarktet, nicht sein Buch.

So bin ich immerhin auf ein, wenn auch bescheidenes, Echo in den Medien gestossen (vgl. Literaturangaben).

Andere Motive? Abschalten vom Alltag, Ausgleich neben dem Beruf?

Nicht eigentlich. Vielleicht Spass daran, für einmal etwas zu produzieren, nicht lediglich etwas abzuwenden. Eine Tätigkeit ohne Verantwortung. Es sei denn, man ist aufgrund abstruser Theorien der Überzeugung, Schriftsteller trügen in irgendeiner Form eine Verantwortung.

Auch eine Arbeit, welche Freiheit belässt. Mehr als sie ein Arzt in sogenannt freier Praxis wohl je wieder haben wird. Vielleicht werden solche Aspekte in Zukunft immer wichtiger, je mehr man als Arzt zu einem gebenchmarkten Funktionär wird.

Also doch eine Art Ausgleich.

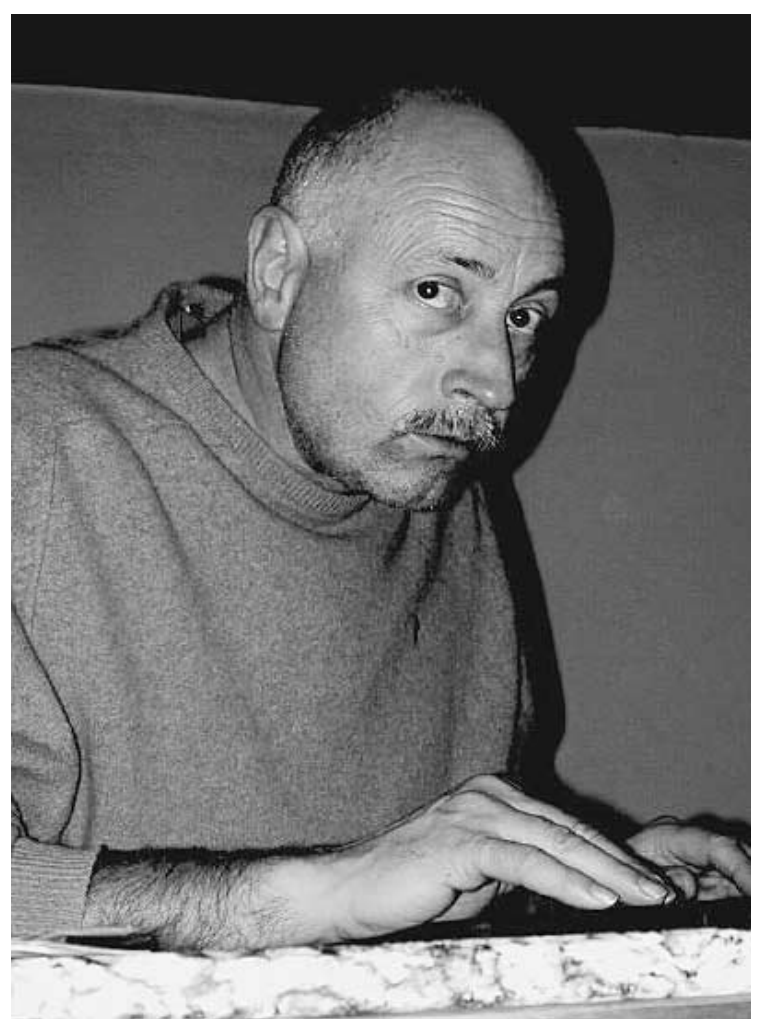

Der nächste Band?

Sollte schon längst erschienen sein. Lässt leider etwas auf sich warten. Noch fehlen 20 Seiten, dann der letzte Schliff und das definitive Cover.

Es braucht schon ordentlich Zeit. Gerade die Passagen, welche erscheinen wie seitenweise belanglos und schludrig hingeschmissen, sind oft am aufwendigsten zu bewerkstelligen. Und soll es gelingen, im grossen seichten Gewässer einzelne Stellen mit etwas mehr Tiefe zu plazieren, wird es erst recht schwierig.

Geplant sind sieben Bände. Das ganze Handlungsgerüst steht schon, und von jedem späteren Band habe ich bereits ein paar Dutzend Seiten geschrieben. Jetzt ist alles eine Fleissarbeit, das Zusammenfügen eines Puzzles, mit dem Versuch, immer mehr Spannung aufzubauen.

Mit BOD (books on demand) bin ich mein eigener Verleger. Habe auch beim Umschlag, gestaltet vom Grafiker Peter Auchli, viel mitgeredet. Die Produktionskosten sind niedrig. Vermarktung, Werbung, Kontakte zur Presse müssen jedoch durch die Autoren selbst vorgenommen werden.

Schwierig ist es zur Zeit noch in der Schweiz, weil der Buchhandel nur zögerlich mitzieht. Das wird sich jedoch, wie in Deutschland, mit Sicherheit in nächster Zukunft ändern.

In Zusammenarbeit mit dem Schweizer Buchzentrum (SBZ) baut BOD Schweiz die notwendigen Strukturen gegenwärtig auf.
Dr. med. Edgar Mosimann

Le Vieux-Moulin

CH-1585 Salavaux

E-Mail: real-touch@bluemail.ch 
Literarische Vorbilder?

Keine. Ich möchte ja niemanden beleidigen. Einen Lieblingsschriftsteller schon: Hans Erich Nossack.

Der hat so Sätze geschrieben wie: «Es war nämlich die Erkenntnis, dass man mir alles, was man mir beigebracht hatte, verkehrt beigebracht hatte."

Hat sehr viel Aktualität, Nossack, und ist meiner Ansicht nach viel zu wenig bekannt, in einer Zeit, in welcher intelligente Menschen sich zunehmend von Systemen erdrückt fühlen, und sich mehr und mehr wieder marginalisieren.

Die Frage des Pseudonyms.

Hat sich natürlich gestellt. Ich hätte dann die ständig vermuteten Querverbindungen zu meiner Person weitgehend unterbunden. Sagen wir mal: ich hätte mehr Narrenfreiheit erhalten. Ich habe «mosimann" gewählt, weil ich mich mit meiner Person hinter die Produktion stellen und nicht verstecken will. Andererseits soll mit dem Weglassen des Vornamens und der Kleinschreibung gewissermassen der Eindruck eines Labels erweckt werden, bei welchem durchaus auch andere Leute mitarbeiten, zum Beispiel eben der Grafiker Peter Auchli, und später die fiktiven Schreibangestellten Sulser und Wülser, oder auch die sich einschaltenden Leser, von welchen es tatsächlich viel mehr hat, als in skeptischen Presseberichten vermutet worden ist.

Einen Wunsch an die Leser der Schweizerischen Ärztezeitung?

Eigentlich nicht.

Oder vielleicht den: Lesen Sie vor dem Einschlafen ausser den Schriften zum TARMED doch gelegentlich wieder einmal ein unterhaltendes Buch.

Zum Beispiel eines aus der Serie SCHUNDROMAN von mosimann.

Nächste Veranstaltungen

- B0D-Lesung, Libromania, Länggass-Strasse 12, Bern, 13. März, 2002, 19.00.

- Literaturwochenende im Maderanertal, 15./16. Juni, 2002.

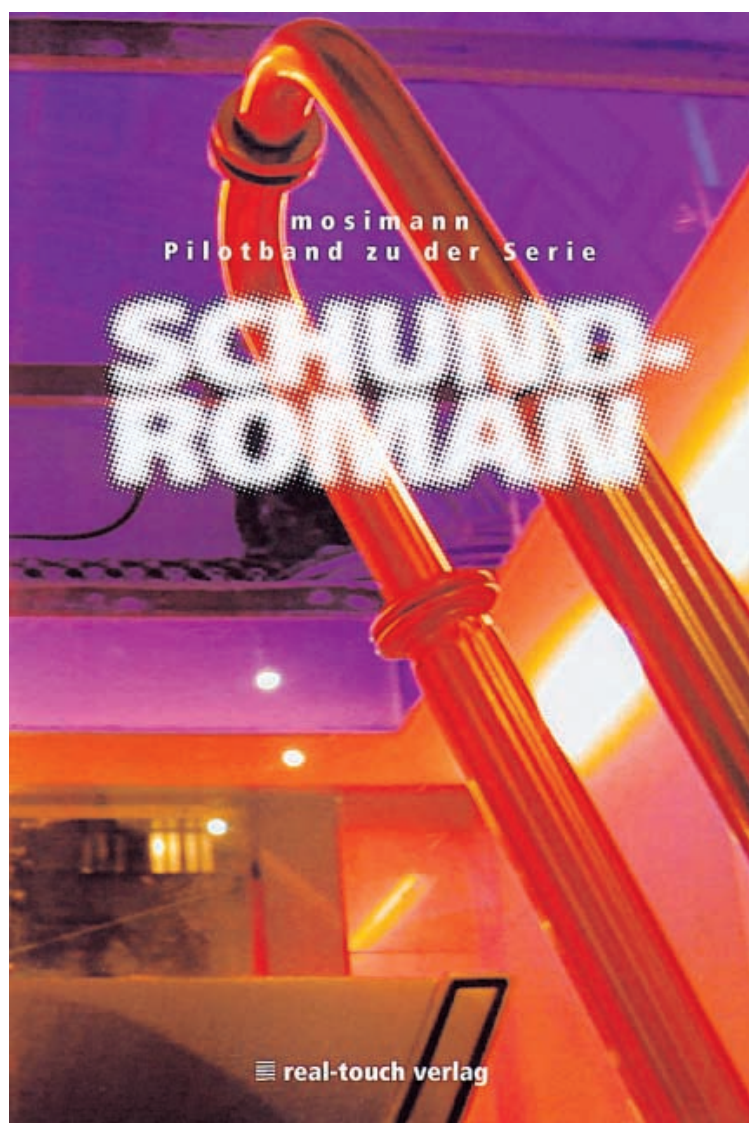

\section{Literatur}

- Binggeli S. Herzchirurg schreibt Schundroman. Annabelle 2001;13.

- Reich M. Herzchirurg schreibt Schundroman. GlücksPost 2001;27.

- Lerch H. Mosimann: «Schundroman». BrunneZytig 3.7.2001.

- Keller P. Über die Kunst, einen Schundroman zu schreiben. Le Lac $2001 ; 7$.

- Schorre A. Das ist eine ultimativ seichte Lektüre. BernerZeitung 8.9.2001.

- Schläpfer F. Ein Friedhof der verlorenen Dichter. Tagesanzeiger 27.12.2001. 